\title{
ON THE CONVERGENCE OF A SEQUENCE OF COMPLETELY POSITIVE MAPS TO THE IDENTITY
}

\author{
GEORGE A. ELLIOTT
}

(Received 26 April 1999; revised 28 September 1999)

\author{
Communicated by G. Robertson
}

\begin{abstract}
It is shown that a sequence of completely positive linear maps on a $\mathrm{W}^{*}$-algebra that converges pointwise in norm to the identity converges uniformly.

2000 Mathematics subject classification: primary $46 \mathrm{~L} 05$.
\end{abstract}

Results closely related to the result of this note, either as important special cases, or in other ways, have been obtained by a number of authors.

In [8-10], Kallman showed that a one-parameter group of automorphisms of a $\mathrm{W}^{*}$-algebra that is continuous with respect to pointwise convergence (in norm) is continuous with respect to uniform convergence.

Underlying Kallman's result is the fact, pointed out in [5] (see also [6, Section 3]), that pointwise and uniform convergence to the identity are the same for any sequence of automorphisms of a $\mathrm{W}^{*}$-algebra.

In [11], Kishimoto and Robinson showed that, in the case of an abelian $W^{*}$-algebra, pointwise and uniform convergence to the identity are the same for a sequence of arbitrary positive linear maps.

The next step was taken by Coulhon and Raynaud, who considered in [4] the case of a properly infinite $\mathrm{W}^{*}$-algebra, and showed that pointwise and uniform convergence to the identity are the same for a sequence of completely positive linear maps. In an earlier article, [7], Groh had asserted this result for weak* continuous completely positive maps, but, as pointed out in [4], his proof contains an error (an incorrect application of the Baire category theorem), which it is not clear how to repair. Coulhon and Raynaud do attribute one of the ideas of their proof to Groh, connected with the particular way

(C) 2000 Australian Mathematical Society $0263-6115 / 2000 \$ A 2.00+0.00$ 
in which complete positivity is used. (Actually, all that they use is the Choi-Schwarz inequality, which holds for 2-positive maps; see [12, Corollary 2.8].)

The purpose of the present note is to extend these results to the missing case, namely, the case that the algebra is finite but not abelian. What is needed is a minor modification of the argument of Coulhon and Raynaud, together with an application of the result of Kishimoto and Robinson (to deal with the type I case).

It should be mentioned that, while Groh proposed in [7] a possible method for reducing the finite case to the infinite, at least for weak* continuous maps-namely, tensoring with the identity map on a factor of type $I_{\infty}$ - his argument to show that pointwise convergence in norm is preserved under tensoring with the identity is not correct, and it does not appear to be possible to complete it. It happens that the assertion itself is correct, at least for sequences (of weak ${ }^{*}$ continuous completely positive maps), but this can only be seen as a consequence of the results of the present note.

While the proof of Kishimoto and Robinson does not extend to the case of a quotient of a $\mathrm{W}^{*}$-algebra, a different proof, given by Coulhon in [3] and by Lotz in [12], does extend in this way. As a result-as the rest of the methods pass to quotients-the full result holds for any quotient of a $\mathrm{W}^{*}$-algebra (or $\mathrm{AW}^{*}$-algebra). (As was shown for automorphisms in [6].)

THEOREM 1. Let $A$ be an $\mathrm{AW}^{*}$-algebra, or the quotient of an $\mathrm{AW}^{*}$-algebra by $a$ closed two-sided ideal. Let $\left(\varphi_{n}\right)$ be a sequence of completely positive linear maps on A such that

$$
\left\|\varphi_{n}(a)-a\right\| \rightarrow 0, \quad a \in A
$$

It follows that

$$
\left\|\varphi_{n}-1\right\| \rightarrow 0
$$

PROOF. The proof follows the proof for automorphisms given in [5] (see also [6]) even more closely than does the proof given in [4], for completely positive maps in the properly infinite case.

The basic step in the proof is to note (see Corollary 4 below) that if

$$
\left\|\left(p \varphi_{n} p-1\right) \mid p A p\right\| \rightarrow 0
$$

for some projection $p \in A$, where $p \varphi_{n} p$ denotes the map $a \mapsto p \varphi_{n}(a) p$, and if $q$ is a projection which is the sum of finitely many orthogonal projections each equivalent to $p \multimap$ or to a subprojection of $p-$, then

$$
\left\|\left(\varphi_{n}-1\right) \mid q A q\right\| \rightarrow 0 .
$$


(In [5] and [6] this implication was established for automorphisms, and in [4] it was established in the case that $q$ is equivalent to $p$. In neither these cases nor the present case does the proof use any special properties of the $\mathrm{C}^{*}$-algebra $A$.)

The proof of the theorem is slightly simpler in the case that $A$ is a quotient of a finite direct $\operatorname{sum} B$ of finite matrix algebras over abelian $\mathrm{AW}^{*}$-algebras. In this case, there exists by hypothesis a projection $p \in A$ with the property mentioned above, with $q=1$, namely, the image in $A$ of an abelian projection of $B$ of central support one. The property in question, that

$$
\left\|\left(p \varphi_{n} p-1\right) \mid p A p\right\| \rightarrow 0
$$

follows from Lemma 5 below, as $p A p$ is a quotient of an abelian $\mathrm{AW}^{*}$-algebra. (Note that Lemma 5 is a special case of the result of [3] and [12]; if the ideal is zero, that is to say, if $p A p$ is an abelian $\mathrm{AW}^{*}$-algebra, the proof of Proposition A1 of [11] is valid.) Hence, $\left\|\varphi_{n}-1\right\| \rightarrow 0$.

In the case that $A$ does not have the above form-that $A$ is the quotient of an $\mathrm{AW}^{*}$-algebra $B$ which is not a finite direct sum of $\mathrm{AW}^{*}$-algebras of type $\mathrm{I}_{n}$ for various finite numbers $n$-there exists an infinite orthogonal sequence $\left(p_{k}\right)$ of projections such that, for each $k$, there exist finitely many orthogonal projections in $A$, equivalent to subprojections of $p_{k}$, with sum a central projection $q_{k}$ in $A$ such that $\left(1-q_{k}\right) A$ belongs to the previous case. Thus, for each $k$,

$$
\left\|\left(\varphi_{n}-1\right) \mid\left(1-q_{k}\right) A\right\| \rightarrow 0 .
$$

Furthermore, if for some $k$,

$$
\left\|\left(\varphi_{n}-1\right) \mid p_{k} A p_{k}\right\| \rightarrow 0
$$

then (by Corollary 4)

$$
\left\|\left(\varphi_{n}-1\right) \mid q_{k} A\right\| \rightarrow 0
$$

and so

$$
\left\|\varphi_{n}-1\right\| \rightarrow 0
$$

as desired. If, on the contrary, this holds for no $k$, then for some $\varepsilon>0$, for every $k$ there exists $n_{k}$ and a projection $f_{k} \in p_{k} A p_{k}$ with

$$
\left\|\varphi_{n_{k}}\left(f_{k}\right)-f_{k}\right\| \geq \varepsilon
$$

As in the proof of Lemma 5, below, this contradicts Lemma 6. (The sequence $\left(f_{k}\right)$ is the image of an orthogonal sequence $\left(p_{k}\right)$ of projections in $B$ under the canonical map $\pi: B \rightarrow A$, and with $\theta_{n}=\pi\left(\varphi_{n}-1\right)$, we have $\theta_{n}\left(\sum_{k \in K} p_{k}\right) \rightarrow 0$ for every $K \subseteq\{1,2, \ldots\}$, while $\left\|\theta_{n_{k}} p_{k}\right\| \geq \varepsilon$.) 
LEMMA $2([2,4])$. Let $A$ be a $\mathrm{C}^{*}$-algebra, and let $\varphi: A \rightarrow A$ be a positive linear map satisfying the Choi-Schwarz inequality,

$$
\varphi(a) \varphi(a)^{*} \leq\|\varphi\| \varphi\left(a a^{*}\right), \quad a \in A .
$$

If $b \in A$ is such that

$$
\varphi(b) \varphi(b)^{*}=\|\varphi\| \varphi\left(b b^{*}\right)
$$

then

$$
\varphi(b) \varphi(a)=\|\varphi\| \varphi(b a), \quad a \in A .
$$

PROOF. This is [4, Lemma 2]-and [2, Corollary 2.8] in the case that $\varphi$ is 2 positive.

COROLLARY 3 (compare [4-6]). Let $A$ be a $\mathrm{C}^{*}$-algebra, and let $\varphi: A \rightarrow A$ be a positive linear map of norm one satisfying the Choi-Schwarz inequality.

If $\varphi$ is equal to the identity on $p A p$ for some projection $p \in A$, and if $q$ is a projection which is the sum of finitely many (orthogonal) projections equivalent to $p-o r$ to subprojections of $p$-by means of partial isometries which, together with their range and support projections, are fixed by $\varphi$, then $\varphi$ is equal to the identity on $q A q$.

The hypothesis $(\varphi-1) \mid p A p=0$ holds if (and only if) $\varphi(p)=p$ and $(p \varphi p-1) \mid$ $p A p=0$, where $p \varphi p$ denotes the map $a \mapsto p \varphi(a) p$.

PROOF. If $x \in q A q$ then by hypothesis

$$
x=\sum b_{i} y_{i j} b_{j}^{*}
$$

where, for every $i$ and $j$, the elements

$$
b_{i}, \quad y_{i j}, \quad b_{j}^{*}, \quad b_{i} b_{i}^{*}, \quad \text { and } \quad b_{j}^{*} b_{j}
$$

are fixed by $\varphi$. In particular, each $b_{i}$ and each $b_{j}^{*}$ fulfills the hypothesis of Lemma 2 above in place of $b$. Hence by Lemma 2 ,

$$
\varphi(x)=\sum \varphi\left(b_{i} y_{i j} b_{j}^{*}\right)=\sum \varphi\left(b_{i}\right) \varphi\left(y_{i j}\right) \varphi\left(b_{j}^{*}\right)=\sum b_{i} y_{i j} b_{j}^{*}=x .
$$

COROLLARY 4 (compare [4-6]). Let A be a unital $\mathrm{C}^{*}$-algebra, and let $\left(\varphi_{n}\right)$ be a sequence of positive linear maps satisfying the Choi-Schwarz inequality. Suppose that $\varphi_{n}$ converges pointwise to the identity:

$$
\left(\varphi_{n}-1\right)(a) \rightarrow 0, \quad a \in A .
$$


Suppose that $q$ is a projection which is the sum of finitely many (orthogonal) projections equivalent to $p$ or to subprojections of $p$. Then if

$$
\left\|\left(p \varphi_{n} p-1\right) \mid p A p\right\| \rightarrow 0,
$$

where $p \varphi_{n} p$ denotes the map $a \mapsto p \varphi_{n} p$ as above, it follows that

$$
\left\|\left(\varphi_{n}-1\right) \mid q A q\right\| \rightarrow 0 .
$$

PROOF. This follows from Corollary 3 applied to the $C^{*}$-algebra $B$ of bounded sequences of elements of $A$ modulo null sequences, and the map $\varphi$ taking the sequence $\left(a_{n}\right)$ (modulo null sequences) to the sequence $\left(\varphi_{n}\left(a_{n}\right)\right)$, and the projections consisting of the constant sequences $(p, p, \ldots)$ and $(q, q, \ldots)$. Note that any constant sequence-in particular corresponding to a fixed projection or partial isometry in $A$-is fixed by $\varphi$ when considered as an element of $B$. In particular, $\varphi$ fixes the unit of $B$, and so has norm one, as is required in order to apply Corollary 3 .

LEMMA $5([11,3,12])$. Let $A$ be the quotient of an abelian $\mathrm{AW}^{*}$-algebra by a closed two-sided ideal. Let $\left(\varphi_{n}\right)$ be a sequence of positive linear maps on $A$ such that

$$
\varphi_{n} a-a \rightarrow 0, \quad a \in A .
$$

It follows that

$$
\left\|\varphi_{n}-1\right\| \rightarrow 0
$$

PROOF. We give a proof which is valid for quotients (unlike that of [11]) which is different from that of [3] and [12]. It is sufficient to prove that

$$
\sup \left\{\left\|\varphi_{n} e-e\right\| ; e \text { a projection in } A\right\} \rightarrow 0 .
$$

Suppose the contrary. Then, for some $\varepsilon>0$, after passing to a subsequence of $\left(\varphi_{n}\right)$, we may choose projections $e_{1}, e_{2}, \ldots$ in $A$ such that

$$
\left\|\varphi_{n} e_{n}-e_{n}\right\| \geq \varepsilon, \quad n=1,2, \ldots
$$

If the projections $e_{1}, e_{2}, \ldots$ are mutually orthogonal, then we obtain a contradiction by [5, Lemma 2], reproduced as Lemma 6 below.

Let us complete the proof by modifying the projections $e_{1}, e_{2}, \ldots$ to be orthogonal. First, since $\varphi_{n}(1)-1 \rightarrow 0$, we may suppose that, as well as $\left\|\varphi_{1} e_{1}-e_{1}\right\| \geq \varepsilon$, also

$$
\left\|\varphi_{1}\left(1-e_{1}\right)-\left(1-e_{1}\right)\right\| \geq \frac{\varepsilon}{2} .
$$


Second, since $\varphi_{n}\left(e_{1}\right)-e_{1} \rightarrow 0$, applying Lemma 2 to the algebra of bounded sequences in $A$ modulo null sequences, as in the proof of Corollary 4 , we see that

$$
\varphi_{n}\left(e_{1} e_{n}\right)-e_{1} \varphi_{n}\left(e_{n}\right) \rightarrow 0,
$$

and so, changing notation, we may suppose that

$$
\left\|\varphi_{n}\left(e_{1} e_{n}\right)-e_{1} \varphi_{n}\left(e_{n}\right)\right\| \leq \frac{\varepsilon}{4}
$$

for all $n \geq 2$. Changing notation a second time, we may suppose also that

$$
\left\|\varphi_{n}\left(\left(1-e_{1}\right) e_{n}\right)-\left(1-e_{1}\right) \varphi_{n}\left(e_{n}\right)\right\| \leq \frac{\varepsilon}{4}, \quad n \geq 2 .
$$

Since, for every $n$,

$$
\max \left(\left\|e_{1}\left(\varphi_{n}\left(e_{n}\right)-e_{n}\right)\right\|,\left\|\left(1-e_{1}\right)\left(\varphi_{n}\left(e_{n}\right)-e_{n}\right)\right\|\right)=\left\|\varphi_{n}\left(e_{n}\right)-e_{n}\right\| \geq \varepsilon,
$$

it follows that, for each $n \geq 2$,

$$
\max \left(\left\|\varphi_{n}\left(e_{1} e_{n}\right)-e_{1} e_{n}\right\|,\left\|\varphi_{n}\left(\left(1-e_{1}\right) e_{n}\right)-\left(1-e_{1}\right) e_{n}\right\|\right) \geq \frac{3 \varepsilon}{4}
$$

Hence, with $f_{1}$ equal to either $e_{1}$ or $1-e_{1}$, as appropriate, and after passing to a subsequence of $\left(\varphi_{n}\right)_{n \geq 2}$ and $\left(e_{n}\right)_{n \geq 2}$ (the same subsequence), we have

$$
\begin{aligned}
\left\|\varphi_{n} f_{1}-f_{1}\right\| & \geq \frac{\varepsilon}{2}, \\
\left\|\varphi_{n}\left(\left(1-f_{1}\right) e_{n}\right)-\left(1-f_{1}\right) e_{n}\right\| & \geq \frac{3 \varepsilon}{4}, \quad n \geq 2 .
\end{aligned}
$$

The third and subsequent steps are similar. In fact, considering instead of $\varphi_{n}$ for $n \geq 2$ the map

$$
\left(1-f_{1}\right) \varphi_{n} \mid\left(1-f_{1}\right) A:\left(1-f_{1}\right) a \mapsto\left(1-f_{1}\right) \varphi_{n}\left(\left(1-f_{1}\right) a\right),
$$

and instead of $e_{2}, e_{3}, \ldots$ the projections $\left(1-f_{1}\right) e_{2},\left(1-f_{1}\right) e_{3}, \ldots$, we may, working inside $\left(1-f_{1}\right) A$, in exactly the same way pass to a subsequence such that, with $f_{2}$ equal to $\left(1-f_{1}\right) e_{2}$ or $\left(1-f_{1}\right)-\left(1-f_{1}\right) e_{2}$, whichever is appropriate, so that in particular $f_{1} f_{2}=0$, we have, as above,

$$
\begin{aligned}
\left\|\left(1-f_{1}\right) \varphi_{2}\left(f_{2}\right)-f_{2}\right\| & \geq \frac{\varepsilon}{2}, \\
\left\|\left(1-f_{1}\right) \varphi_{n}\left(\left(1-f_{1}-f_{2}\right) e_{n}\right)-\left(1-f_{1}-f_{2}\right) e_{n}\right\| & \geq \frac{3 \varepsilon}{4}, \quad n \geq 3,
\end{aligned}
$$


and so also

$$
\begin{aligned}
\left\|\varphi_{2}\left(f_{2}\right)-f_{2}\right\| & \geq \frac{\varepsilon}{2}, \\
\left\|\varphi_{n}\left(\left(1-f_{1}-f_{2}\right) e_{n}\right)-\left(1-f_{1}-f_{2}\right) e_{n}\right\| & \geq \frac{3 \varepsilon}{4}, \quad n \geq 3 .
\end{aligned}
$$

This procedure may be continued.

In this way, we obtain (passing to a subsequence of $\left(\varphi_{n}\right)$ and modifying the original choice of the sequence $\left.\left(e_{n}\right)\right)$ an orthogonal sequence of projections $\left(f_{n}\right)$ in $A$ such that

$$
\left\|\varphi_{n} f_{n}-f_{n}\right\| \geq \frac{\varepsilon}{2}
$$

As mentioned above, this contradicts Lemma 6. (If $A$ is a quotient of the AW*-algebra $B$, then, with $\pi$ the canonical mapping $B \rightarrow A$, and $\left(e_{n}\right)$ an orthogonal sequence of projections in $B$ with $\pi\left(e_{n}\right)=f_{n}$, and with $\theta_{n}=\pi\left(\varphi_{n}-1\right)$, we have

$$
\theta_{n}\left(\sum_{k \in K} e_{k}\right) \rightarrow 0
$$

for every $K \subseteq\{1,2, \ldots\}$, but $\left\|\theta_{n} e_{n}\right\| \geq \varepsilon / 2$ for all $n$, in direct contradiction with Lemma 6.)

LEMMA 6 ([5, Lemma 2]). Let $B$ be an $\mathrm{AW}^{*}$-algebra, and let $\left(e_{n}\right)$ be an orthogonal sequence of projections in $B$. Let $\left(\theta_{n}\right)$ be a bounded sequence of linear maps from $A$ into a normed linear space such that for every $K \subseteq\{1,2, \ldots\}$,

$$
\theta_{n}\left(\sum_{k \in K} e_{k}\right) \rightarrow 0
$$

Then

$$
\sup _{k}\left\|\theta_{n}\left(e_{k}\right)\right\| \rightarrow 0
$$

REMARKS. It is not clear for exactly which class of $\mathrm{C}^{*}$-algebras the two types of convergence of a sequence of completely positive maps towards the identity coincideor, for such $\mathrm{C}^{*}$-algebras, for exactly which sequences of maps the two notions of convergence coincide.

For instance, in the commutative case, as showed in [3] and [12], this holds more generally than in an abelian $\mathrm{AW}^{*}$-algebra (or a quotient of one). In particular (as the proof of Lemma 5 shows) it holds in an abelian $\aleph_{0}-\mathrm{AW}^{*}$-algebra (or quotient of one). The absence of a suitable type classification of an $\aleph_{0}-\mathrm{AW}^{*}$-algebra in the non-abelian case, however, makes the choice of projections $p_{k}$ as in the proof of Theorem 1 problematic. (The algebra may even fail to have a unit.) 
The proof does have in common with the proof in [4] in the properly infinite case that it only uses the Choi-Schwarz inequality, rather than the full force of complete positivity. Whether (possibly using the Kadison Schwarz inequality rather than that of Choi) it can be extended to deal with arbitrary positive maps-even Jordan automorphisms-is not clear.

As far as more general sequences of maps are concerned-rather than $\left(\varphi_{n}-1\right)$ where $\varphi_{n}$ is some kind of positive map-the following dilatory comments may be made. For a sequence $\left(\varphi_{n}-1\right)$ where each $\varphi_{n}$ is a complete contraction, or even just a 2-contraction, the same proof is applicable, as Corollary 4 also holds for such mapswith the same proof (the map $\varphi$ on the asymptotic sequence algebra is a positive map and so by [2, Corollary 2.8] satisfies the Choi-Schwarz inequality). For a sequence $\left(\varphi_{n}-\psi\right)$ where $\varphi_{n}$ and $\psi$ are automorphisms, the question is equivalent to that in the case $\left(\varphi_{n}-1\right)$, as one may consider instead the sequence $\left(\varphi_{n} \psi^{-1}-1\right)$. If $\varphi_{n}$ and $\psi$ are more general endomorphisms, not necessarily automorphisms or even $*$-algebra maps, the proof of Theorem 1 (or of [5] and [6]) may still be seen to be valid. The case of a sequence $\left(\delta_{n}\right)$ where $\delta_{n}$ is a (bounded) derivation is easily reduced to the automorphism case (and was dealt with directly in [6]). Of course, for an arbitrary sequence of bounded maps $\left(T_{n}\right)$ converging to zero pointwise but not uniformly, on an infinite-dimensional $\mathrm{W}^{*}$-algebra (in fact, as pointed out in [12], on any infinitedimensional Banach space), pointwise and uniform convergence are not the same. (Note, however, that if $T_{n}$ is a weak ${ }^{*}$ continuous linear functional then this is true if - and only if - the $\mathrm{W}^{*}$-algebra is a direct sum of finite type $\mathrm{I}$ factors. (Use a sliding hump argument.) Furthermore, if $T_{n}=f_{n}-f$ where $f_{n}$ is a state and $f$ is a fixed pure state then the two notions of convergence are known to be the same--[1].)

\section{References}

[1] C. A. Akemann, 'Sequential convergence in the dual of a W*-algebra', Comm. Math. Phys. 7 (1968), 222-224.

[2] M.-D. Choi, 'A Schwarz inequality for positive linear maps on $C^{*}$-algebras', Illinois J. Math. 18 (1974), 565-574.

[3] T. Coulhon, 'Suites d'opérateurs sur un espace $\mathscr{C}(K)$ de Grothendieck', C. R. Acad. Sci. Paris Sér. I Math. 298 (1984), 13-15.

[4] T. Coulhon and Y. Raynaud, 'Convergence vers l'identité de suites d'opérateurs définis sur $\mathscr{L}(H)$ ', C. R. Acad. Sci. Paris Sér. I Math. 301 (1985), 111-113.

[5] G. A. Elliott, 'Convergence of automorphisms in certain C*-algebras', J. Funct. Anal. 16 (1972), 204-206.

[6] — ' 'On derivations of AW*-algebras', Tôhoku Math. J. 30 (1978), 263-276.

[7] U. Groh, 'Norm continuity of strongly continuous semigroups on $\mathrm{W}^{*}$-algebras', in: Semesterbericht Funktionalanalysis Tübingen, Wintersemester 1983-1984. 
[8] R. R. Kallman, 'Uniform continuity, unitary groups, and compact operators', J. Funct. Anal. 1 (1967), 245-253.

[9] — , 'Unitary groups and automorphisms of operator algebras', Amer. J. Math. 91 (1969), 785-806.

[10] - 'One-parameter groups of *-automorphisms of $\mathrm{II}_{1}$ von Neumann algebras', Proc. Amer. Math. Soc. 24 (1970), 336-340.

[11] A. Kishimoto and D. W. Robinson, 'Subordinate semigroups and order properties', J. Austral. Math. Soc. Ser. A 31 (1981), 59-76.

[12] W. P. Lotz, 'Uniform convergence of operators on $\mathrm{L}^{\infty}$ and similar spaces', Math. Zeit. 190 (1985), $207-220$.

Department of Mathematics

University of Toronto

Toronto

Canada M5S 3G3

Department of Mathematics

University of Copenhagen

Copenhagen

Denmark

and

The Fields Institute

222 College Street

Toronto

Canada M5T 3J1

e-mail: elliott@math.utoronto.ca 